\title{
Floristic features of Damietta area in the north east Nile Delta, Egypt.
}

\author{
Ibrahem, A. Mashaly \\ El-Said, F. El Halawany \\ and
}

\author{
Gehan Omar \\ Botany Department, Faculty of Science, Mansoura University, Egypt
}

Mashaly, I.A., El-Halawany, E.F. \& Omar, G. 2002. Floristic features of Damietta area in the north east Nile Delta, Egypt. Taeckholmia 22(1):101-114.

Floristic features of three habitat types in the Damietta Governorate namely: field crops, orchards and canal banks are described. The study habitats include 171 species related to 128 genera, grouped under 44 families with Gramineae attaining 36 species (21.05\%), Compositae 21 species (12.28\%) and Chenopodiaceae 15 species (8.77\%), these are the main families. The recorded species comprise 98 annual species (57.31\%), 8 biennial species (4.68\%) and 65 perennial species (38.01\%). The annual species are classified under three categories as follows: a) all year active annuals (14 species, b) winter-spring active annuals (64 species) and c) summer-autumn active annuals (20 species).

According to the life-form spectrum the recorded species are classified into: therophytes $(60.23 \%)$ cryptophytes (22.22\%), chamaeophytes (7.60\%), hemicryptophytes (7.02\%), nanophanerophytes (1.75\%) and parasites $(1.17 \%)$

The floristic analysis of the study area reveals that, the Mediterranean elements (mono, bi - pluriregional) include 81 taxa (47.36\%), Cosmopolitan element 28 species (16.37\%), Palaeotropical 22 species (12.68\%) and Pantropical 21 species (12.28\%). These represent the major chorotypes. The other floristic categories are poorly represented. This indicates that, the study area is obviously belong to the Mediterranean Territory with the enterance of other elements to the area.

Key wards: Chorology, Damietta, Egypt, flora, habitats, life forms.

\section{Introduction}

The terms weedes, weed flora and weed vegetation are commonly used in the literature concerned with Egyptian flora. The Egyptian weed flora is charactristic to man-made habitats such as cultivated fields, gardens, palm groves, orchards, lawns, roads, road sides, canal banks, canal, channels, ditches, drains, etc. (Kosinova', 1974a).

Earlier works on the weed flora in field crops, orchards, canals and canal banks in Nile Delta one few, among which to mention Simpson (1932), Imam and Kosinova (1972) and Kosinova $(1974,1975)$. Works during the last decade include those of, Abd El-Ghani and Amer (1990) who studied the weed assemblages in broad bean fields in Monofiya Governorate, Shaltout and El-Fahar (1991) who studied the diversity and phenology of weed communities in the Nile Delta. Zaki and Mashaly (1992) studied the seasonal aspects of the flora of the field crops in Mansoura District. The species richness and phenology of vegetation along irrigation canals and drains in the Nile Delta were studied by Shaltout et al. (1994). Shalaby (1995) described the floristic features in field crops and canal banks in Kafr ElSheikh Governorate. El-Demerdash et al. (1997) studied the weed flora associated with field crops and orchards in Dakahleya Governorate. El-Kady et al. (1999), describes the variation in the weed flora associated with twelve common orchards in the middle Delta region. The weed flora of Date palm orchards were studied in the northwest of the Nile Delta by El-Fahar 
(2000) and El-Halawany (2000) respectively. Sheded and Turki (2000) studied the weed flora associated with field crops and orchards in Menofiya governorate. The present investigation aims at studying the floristic composition in the area of Damietta Province to analyse its flora and to detect the taxonomic, geographic and chorological significance of its components.

\section{Study Area}

Damietta district is located in the extreme part of the Damietta branch of the Nile, north east Nile Delta (Fig. 1). The total agriculture area of this district is 115892 feddans. Out of this area, 109356 feddans is cultivated with field crops and 6536 feddans is cultivated with orchards. The study area is influenced by the Mediterranean climatic conditions. According to Ayyad et al. (1983), this area lies in the attenuated arid province characterized by a short dry period, with warm summer $\left(20-34.5^{\circ} \mathrm{C}\right)$, mild winter $\left(10-20^{\circ} \mathrm{C}\right)$ with an aridity index $(\mathrm{P} / \mathrm{ETP})$ less than 0.03 , where $\mathrm{P}$ is the precipitation and ETP is the potential evapotranspiration according to Penman's formula. The mean relative humidity in the area is $66.5 \%$. The rainfall was $(100 \mathrm{~mm} / \mathrm{y})$ during winter season.

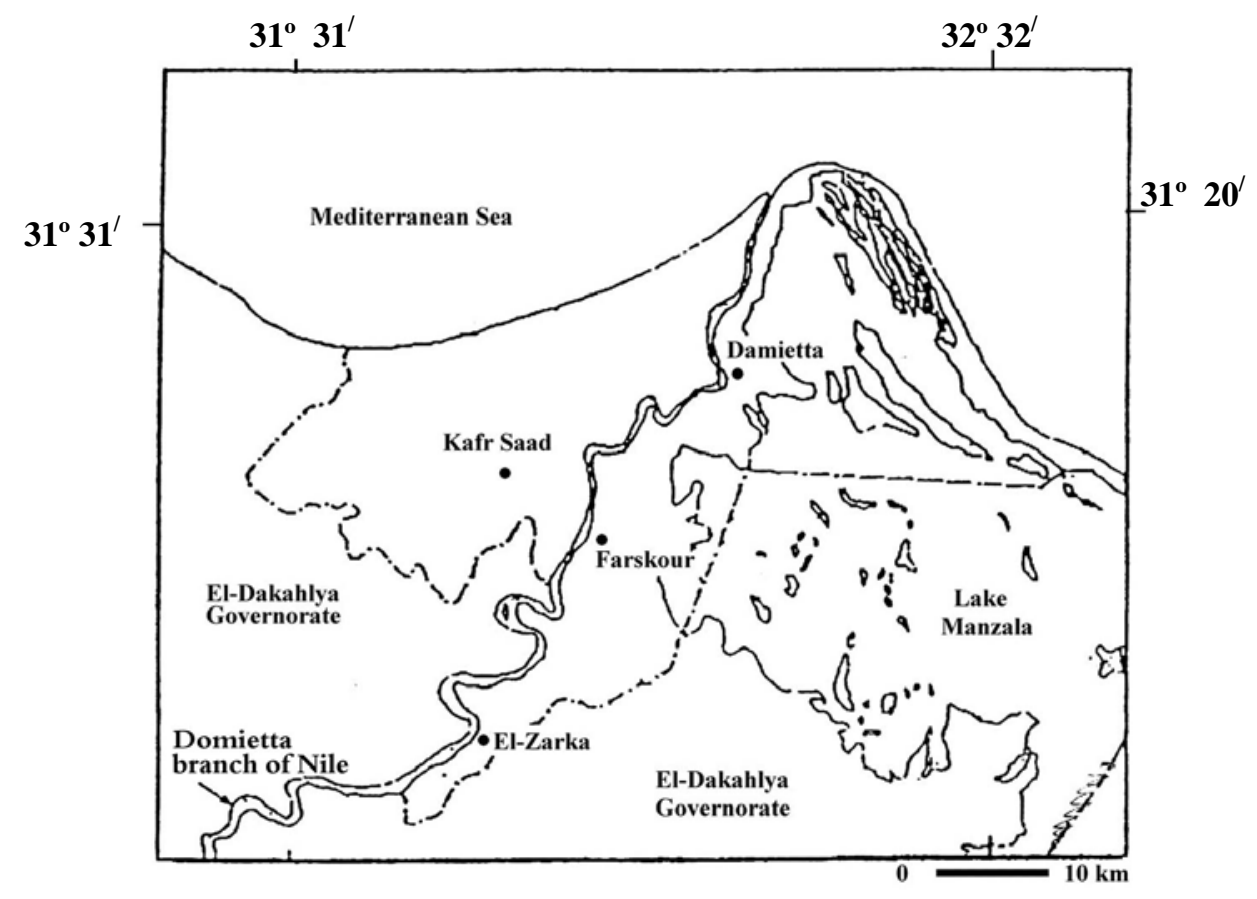

Fig.1. Map of Damietta Governorate showing different sites $(\bullet)$ of the study area. 
Floristic features of Damietta area in the north east Nile Delta, Egypt

\section{Methods}

Between 1999 - 2000, extensive field trips were carried out to the study area. Sampling sites were selected to cover the major agricultural sectors of Damietta district: Damietta, Kafr Saad, Faraskour and El-Zarka. (Fig. 1). The major field crops selected for this study were: wheat (Triticum aestivum), clover (Trifolium alexandrinum), broad bean (Vicia faba) as winter crops and maize (Zea mays), cotton (Gossypium barbadense) and rice (Oryza sativa) as summer crops. Fields of summer and winter vegetables were also surveyed. In orchards, were raised grape (Vitis vinifera) and gugava (Psidium guijava). Furthermore, canal bank plants have been recorded in each locality during the regular visits. Identification and nomenclature of plants followed Tackholm (1974) and Boulos (1995). The description and classification of life forms are due to Raunkiaer (1934). Analysis of phytogeographical ranges are carried out according to Tutin et al. (1964-1980), Zohary (1966 \& 1972), Davis (1965-1985) and Meikle (1977 \& 1985). Voucher specimens were deposited in the Herbarium of Botany Department, Faculty of Science, Mansoura University.

\section{Results}

\section{Floristic records}

The records of weed assemblages in the different habitats under investigation are summed in terms of presence estimates (P\%). Table (1) present the floristic composition of three habitats namely: canal and canal banks, orchards and field crops in Damietta area. It shows that, the total number of weeds recorded in the study area is 171 species including 65 perennials, 8 biennials and 98 annuals. The perennials comprise 12 species recorded in all habitats $(\mathrm{p}=100 \%), 14$ species recorded in two habitats $(\mathrm{P}=66.66 \%)$ and 39 species recorded in only one habitat $(\mathrm{P}=33.33 \%)$. The biennials include one species (Apium graveolens) which is recorded in the three habitats $(\mathrm{P}=100 \%)$, five species recorded in two habitats $(\mathrm{P}=66.66 \%)$ namely: Beta vulgaris, Chenopodium ambrosoides, Rorippa palustris, Sesbania sericea and Spergularia marina: Centaurea calcitrapa and Coronopus squamatus are recorded in one habitat only $(\mathrm{P}=33.33 \%)$. (Table 1):

On the other hand, the annuals (98 species) can be grouped under three categories

a- All year annuals including 14 species recorded in the habitats $(\mathrm{P}=100 \%)$.

b- Winter-spring annuals comprising 64 species are distributed as follows: 19 species recorded in three habitats $(\mathrm{P}=100 \%), 15$ species in two habitats $(\mathrm{P}=66.66 \%)$ and 30 species in only one habitat $(\mathrm{P}=33.33 \%)$.

c- Summer-autumn annuals comprise 20 species are, of which: 10 species were recorded two habitats $(\mathrm{P}=66.66 \%)$ and another 10 species were recorded in one habitat $(\mathrm{P}=$ 33.33\%). 
Table (1). Floristic composition of the different habitat types of the study area.

\begin{tabular}{|c|c|c|c|c|c|c|c|c|}
\hline \multirow[b]{2}{*}{ No } & \multirow[b]{2}{*}{ Species } & \multirow[b]{2}{*}{$\begin{array}{l}\Xi \\
\stackrel{\Xi}{0} \\
\stackrel{\leftrightarrows}{ٌ}\end{array}$} & \multirow[b]{2}{*}{ Chorophyte } & \multicolumn{3}{|c|}{ Habitat type } & \multirow[b]{2}{*}{ 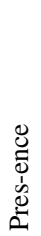 } & \multirow{2}{*}{ 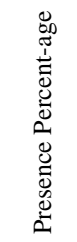 } \\
\hline & & & & 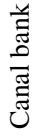 & 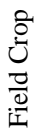 & 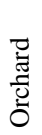 & & \\
\hline \multicolumn{9}{|c|}{ A. Perennials:- } \\
\hline 1 & Alternanthera sessilis (L.) DC. & $\mathrm{He}$ & PAN & + & + & + & 3 & 100.00 \\
\hline 2 & Aster squamatus (Spreng.) Hieron & $\mathrm{CH}$ & NEO & + & + & + & 3 & 100.00 \\
\hline 3 & Cynodon dactylon (L.) Pers. & G & COSM & + & + & + & 3 & 100.00 \\
\hline 4 & Cyperus rotundus L. & G & PAN & + & + & + & 3 & 100.00 \\
\hline 5 & Leptochloa fusca (L.) Kunth & $\mathrm{G}, \mathrm{He}$ & PAL & + & + & + & 3 & 100.00 \\
\hline 6 & Lotus glaber Mill & $\mathrm{H}$ & ER-SR+M+IR-TR & + & + & + & 3 & 100.00 \\
\hline 7 & Paspalum distichum L. & G & PAN & + & + & + & 3 & 100.00 \\
\hline 8 & Phragmites australis (Cav)Trin. ex steud. & $\mathrm{G}, \mathrm{He}$ & COSM & + & + & + & 3 & 100.00 \\
\hline 9 & Phyla nodiflora (L.) Greene & $\mathrm{Ch}$ & PAN & + & + & + & 3 & 100.00 \\
\hline 10 & Plantago major L. & $\mathrm{H}$ & COSM & + & + & + & 3 & 100.00 \\
\hline 11 & Pluchea dioscoridis (L.) DC & $\mathrm{NPh}$ & S-Z + SA-SI & + & + & + & 3 & 100.00 \\
\hline 12 & Polypogon viridis (Gouan) Breistr & $\mathrm{H}$ & $\mathrm{ME}+\mathrm{IR}-\mathrm{TR}+\mathrm{ER}-\mathrm{SR}$ & + & + & + & 3 & 100.00 \\
\hline 13 & Bromus catharticus Vahl & $\mathrm{G}$ & ER-SR+ME+IR-TR & - & + & + & 2 & 66.66 \\
\hline 14 & Convolvulus arvensis L. & $\mathrm{H}$ & COSM & + & + & - & 2 & 66.66 \\
\hline 15 & Cyperus alopecuroides Rottb. & $\mathrm{He}$ & PAN & + & + & - & 2 & 66.66 \\
\hline 16 & Echinochloa stagnina (Retz.) P. Beanv. & $\mathrm{G}, \mathrm{He}$ & PAL & + & + & - & 2 & 66.66 \\
\hline 17 & Imperata cylindrica (L.) Beauv. & $\mathrm{H}$ & PAL & + & - & + & 2 & 66.66 \\
\hline 18 & Leersia hexandra Swartz & $\mathrm{He}$ & PAN & + & + & - & 2 & 66.66 \\
\hline 19 & Lemna gibba L. & Hy & COSM & + & + & - & 2 & 66.66 \\
\hline 20 & Mentha longifolia (L.) Huds. & $\mathrm{He}$ & PAL & + & + & - & 2 & 66.66 \\
\hline 21 & Oxalis corniculata L. & $\mathrm{H}$ & COSM & + & + & - & 2 & 66.66 \\
\hline 22 & Panicum repens L. & G & PAN & + & - & + & 2 & 66.66 \\
\hline 23 & Paspalidium geminatum (Forssk..) Stapf. & $\mathrm{He}$ & PAL & + & - & + & 2 & 66.66 \\
\hline 24 & Persicaria salicifolia Broussex Willd & G & PAL & + & - & + & 2 & 66.66 \\
\hline 25 & Polygonum equisetiforme Sibth. \& Sm. & G & $\mathrm{ME}+\mathrm{IR}-\mathrm{TR}$ & + & - & + & 2 & 66.66 \\
\hline 26 & Sonchus macrocarpus Boulos et. C.Jeffrey & $\mathrm{Ch}$ & Egypt (endemic) & + & - & + & 2 & 66.66 \\
\hline 27 & Adiantum capillus-veneris L. & $\mathrm{He}$ & ME+IR-TR-ER-SR & + & - & - & 1 & 33.33 \\
\hline 28 & Alhagi graecorum Boiss. & $\mathrm{H}$ & PAL & + & - & - & 1 & 33.33 \\
\hline 29 & $\begin{array}{l}\text { Arthrocnemum macrostachyum (Moric.) } \\
\text { Moris et Delponte }\end{array}$ & $\mathrm{Ch}$ & ME+SR-SI & + & - & - & 1 & 33.33 \\
\hline 30 & Arundo donax L. & He, G & Cult. \& Nat. & + & - & - & 1 & 33.33 \\
\hline 31 & Atriplex portulacoides L. & $\mathrm{Ch}$ & $\mathrm{ME}+\mathrm{ER}-\mathrm{SR}+\mathrm{IR}-\mathrm{TR}$ & + & - & - & 1 & 33.33 \\
\hline 32 & Carex extensa Good & $\mathrm{G}$ & ME+ER-SR & + & - & - & 1 & 33.33 \\
\hline 33 & Ceratophyllum demersum L. & Hy & COSM & + & - & - & 1 & 33.33 \\
\hline 34 & Cressa cretica L. & $\mathrm{H}$ & $\mathrm{ME}+\mathrm{PAL}$ & + & - & - & 1 & 33.33 \\
\hline 35 & Cynanchum acutum L. & $\mathrm{H}$ & ME+IR-TR & + & - & - & 1 & 33.33 \\
\hline 36 & Cyperus articulatus L. & $\mathrm{G}, \mathrm{He}$ & PAL & + & - & - & 1 & 33.33 \\
\hline 37 & Cyperus Laevigatus L. & $\mathrm{G}, \mathrm{He}$ & PAL & + & - & - & 1 & 33.33 \\
\hline 38 & Eichhornia crassipes(Mart.) Solms-Laub. & Hy & NEO & + & - & - & 1 & 33.33 \\
\hline 39 & Elymus elongatus (Host) Runemark & $\mathrm{G}$ & $\mathrm{ME}$ & + & - & - & 1 & 33.33 \\
\hline 40 & Halocnemum strobilaceum (Pallas) M.Bieb. & $\mathrm{Ch}$ & $\mathrm{ME}+\mathrm{IR}-\mathrm{TR}+\mathrm{SA}-\mathrm{SI}$ & + & - & - & 1 & 33.33 \\
\hline 41 & Heliotropium curassavicum L. & $\mathrm{Ch}$ & $\mathrm{NEO}$ & + & - & - & 1 & 33.33 \\
\hline 42 & Inula crithmoides L. & $\mathrm{Ch}$ & ME+IR-TR-ER-SR & + & - & - & 1 & 33.33 \\
\hline 43 & Ipomoea carnea Jacq. & $\mathrm{Ch}$ & Cult. \& Nat. & + & - & - & 1 & 33.33 \\
\hline 44 & Juncus acutus L. & $\mathrm{He}$ & $\mathrm{ME}+\mathrm{IR}-\mathrm{TR}+\mathrm{ER}-\mathrm{SR}$ & + & - & - & 1 & 33.33 \\
\hline 45 & Juncus rigidus Desf. & $\mathrm{G}, \mathrm{He}$ & $\mathrm{ME}+\mathrm{SA}-\mathrm{SI}+\mathrm{IR}-\mathrm{TR}$ & + & - & - & 1 & 33.33 \\
\hline 46 & Juncus subulatus Forssk. & $\mathrm{G}, \mathrm{He}$ & $\mathrm{ME}+\mathrm{IR}-\mathrm{TR}+\mathrm{SA}-\mathrm{SI}$ & + & - & - & 1 & 33.33 \\
\hline 47 & Limonium narbonnense Mill & $\mathrm{H}$ & $\mathrm{ME}$ & + & - & - & 1 & 33.33 \\
\hline 48 & Ludwigia stolonifera (Guill et Perr.) Raven & $\mathrm{He}$ & $\mathrm{S}-\mathrm{Z}$ & + & - & - & 1 & 33.33 \\
\hline 49 & Nymphaea lotus L. var. aegyptia Tuzs. & Hy & PAL & + & - & - & 1 & 33.33 \\
\hline
\end{tabular}


Table (1). Cont.

\begin{tabular}{|c|c|c|c|c|c|c|c|c|}
\hline \multirow[b]{2}{*}{ No } & \multirow[b]{2}{*}{ Species } & \multirow[b]{2}{*}{ 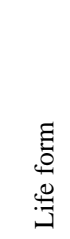 } & \multirow[b]{2}{*}{ Chorophyte } & \multicolumn{3}{|c|}{ Habitat type } & \multirow[b]{2}{*}{ 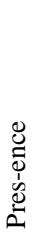 } & \multirow{2}{*}{ 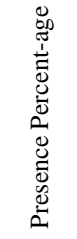 } \\
\hline & & & & 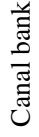 & 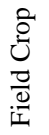 & 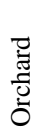 & & \\
\hline 50 & Persicaria lapathifolia Willd. & G & PAL & + & - & - & 1 & 33.33 \\
\hline 51 & Persicaria senegalensis (Meisn) Sojak & G & PAL & + & - & - & 1 & 33.33 \\
\hline 52 & Pistia stratiotes L. & Hy & PAN & + & - & - & 1 & 33.33 \\
\hline 53 & Saccharum spontaneum L. & G.He & $\mathrm{ME}+\mathrm{PAL}$ & + & - & - & 1 & 33.33 \\
\hline 54 & Scirpus litoralis Schrad & G & $\mathrm{ME}+\mathrm{PAL}$ & + & - & - & 1 & 33.33 \\
\hline 55 & Scripus maritimus L. & G & COSM & + & - & - & 1 & 33.33 \\
\hline 56 & Silybum marianum (L.) Gaertn & $\mathrm{H}$ & ME+IR-TR+ER-SR & + & - & - & 1 & 33.33 \\
\hline 57 & Sorghum virgatum (Hack) stapf & G & SA-SI & + & - & - & 1 & 33.33 \\
\hline 58 & Suaeda pruinosa lang. & $\mathrm{Ch}$ & ME & + & - & - & 1 & 33.33 \\
\hline 59 & Suaeda vera Forssk. ex. J.F. Gmelin & $\mathrm{Ch}$ & ME+SA-SI+ER-SR & + & - & - & 1 & 33.33 \\
\hline 60 & Tamarix nilotica (Ehrenb.) Bge. & $\mathrm{NPh}$ & SA-SI+S-Z & + & - & - & 1 & 33.33 \\
\hline 61 & Tamarix tetragyna Ehrenb. & $\mathrm{NPh}$ & SA-SI+ME+IR-TR & + & - & - & 1 & 33.33 \\
\hline 62 & Typha domingensis Pers. & $\mathrm{He}$ & PAN & + & - & - & 1 & 33.33 \\
\hline 63 & Veronica anagallis-aquatica L. & $\mathrm{He}$ & COSM & + & - & - & 1 & 33.33 \\
\hline 64 & Vigna luteola (Jacq.) Benth. & $\mathrm{H}$ & PAL & + & - & - & 1 & 33.33 \\
\hline 65 & Zygophyllum aegyptium A. Hosny & $\mathrm{Ch}$ & ME & + & - & - & 1 & 33.33 \\
\hline \multicolumn{9}{|c|}{ B. Biennials: } \\
\hline 1 & Apium graveolens L. & Th & ME+ER-SR+IR-TR & + & + & + & 3 & 100.00 \\
\hline 2 & Beta vulgaris L. & Th & $\mathrm{ME}+\mathrm{IR}-\mathrm{TR}+\mathrm{ER}-\mathrm{SR}$ & - & + & + & 2 & 66.66 \\
\hline 3 & Chenopodium ambrosioides L. & Th & COSM & + & - & + & 2 & 66.66 \\
\hline 4 & Rorippa palustris (L.) Besser & Th & ME+ER-SR+IR-TR & + & + & - & 2 & 66.66 \\
\hline 5 & Sesbania sericea (Willd.) Link & Th & PAL & + & + & - & 2 & 66.66 \\
\hline 6 & Spergularia marina L. & Th & ER-SR+ME+IR-TR & + & + & - & 2 & 66.66 \\
\hline 7 & Centaurea calcitrapa L. & $\mathrm{Ch}$ & ME+ER-SR & + & - & - & 1 & 33.33 \\
\hline 8 & Coronopus squamatus (Forssk.) Asch. & Th & ME+ER-SR+IR-TR & - & + & - & 1 & 33.33 \\
\hline \multicolumn{9}{|c|}{ C. Annuals: } \\
\hline & a. All year annuals: & & & & & & & \\
\hline 1 & Amoranthus lividus L. & Th & ME+IR-TR & + & + & + & 3 & 100.00 \\
\hline 2 & Chenopodium glaucum L. & Th & $\mathrm{ME}+\mathrm{ER}-\mathrm{SR}$ & + & + & + & 3 & 100.00 \\
\hline 3 & Chenopodium murale L. & Th & COSM & + & + & + & 3 & 100.00 \\
\hline 4 & Conyza bonariensis (L.) Crong. & Th & NEO & + & + & + & 3 & 100.00 \\
\hline 5 & Echinochloa colona (L.) Link & Th & PAN & + & + & + & 3 & 100.00 \\
\hline 6 & Echinochloa cruss-galli (L.) P. Beauv. & Th & PAN & + & + & + & 3 & 100.00 \\
\hline 7 & Gnaphalium luteo-album L. & Th & COSM & + & + & + & 3 & 100.00 \\
\hline 8 & Hibiscus trionum L. & Th & PAL & + & + & + & 3 & 100.00 \\
\hline 9 & Portulaca oleracea L. & Th & COSM & + & + & + & 3 & 100.00 \\
\hline 10 & Rumex dentatus L. & Th & ME+IR-TR+SA-SI & + & + & + & 3 & 100.00 \\
\hline 11 & Solanum nigrum L. & Th & COSM & + & + & + & 3 & 100.00 \\
\hline 12 & Sonchus oleraceus L. & Th & COSM & + & + & + & 3 & 100.00 \\
\hline 13 & Conyza aegyptiaca (L.) Dryand. & Th & ME & - & + & + & 2 & 66.66 \\
\hline 14 & $\begin{array}{l}\text { Ethulia conyzoides L. } \\
\text { b. Winter-spring annuals: }\end{array}$ & Th & PAL & + & + & - & 2 & 66.66 \\
\hline 1 & Anagallis arvensis L. var. arvensis & Th & COSM & + & + & + & 3 & 100.00 \\
\hline 2 & $\begin{array}{l}\text { Anagallis arvensis L. var. caerulea (L.) } \\
\text { Gouan }\end{array}$ & Th & COSM & + & + & + & 3 & 100.00 \\
\hline 3 & Centaurium pulchellum (Swartz) Druce & Th & ME+IR-TR+ER-SR & + & + & + & 3 & 100.00 \\
\hline 4 & Chenopodium album L. & Th & COSM & + & + & + & 3 & 100.00 \\
\hline 5 & Chenopodium ficifolium Sm. & Th & ME-ER-SR & + & + & + & 3 & 100.00 \\
\hline 6 & Cichorium endivia $\mathrm{L}$. & Th & ME+IR-TR & + & + & + & 3 & 100.00 \\
\hline 7 & Coronopus didymus (L.) Sm. & Th & COSM & + & + & + & 3 & 100.00 \\
\hline 8 & Lolium perenne L. & Th & ME+ER-SR+IR-TR & + & + & + & 3 & 100.00 \\
\hline
\end{tabular}


Table (1). Cont.

\begin{tabular}{|c|c|c|c|c|c|c|c|c|}
\hline \multirow[b]{2}{*}{ No } & \multirow[b]{2}{*}{ Species } & \multirow[b]{2}{*}{ 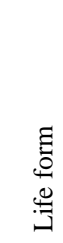 } & \multirow[b]{2}{*}{ Chorophyte } & \multicolumn{3}{|c|}{ Habitat type } & \multirow[b]{2}{*}{ 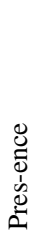 } & \multirow{2}{*}{ 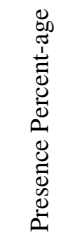 } \\
\hline & & & & 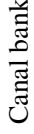 & 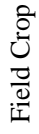 & 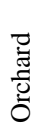 & & \\
\hline 9 & Malva parvifora $\mathrm{L}$. & $\mathrm{Th}$ & ME+IR-TR & + & + & + & 3 & 100.00 \\
\hline 10 & Medicago intertexta (L.) Mill. & Th & $\mathrm{ME}+\mathrm{ER}-\mathrm{SR}$ & + & + & + & 3 & 100.00 \\
\hline 11 & Medicago polymorpha L. & Th & COSM & + & + & + & 3 & 100.00 \\
\hline 12 & Melilotus indicus (L.) All. & Th & ME+IR-TR+SA-SI & + & + & + & 3 & 100.00 \\
\hline 13 & Phalaris minor Retz. & Th & ME+IR-TR & + & + & + & 3 & 100.00 \\
\hline 14 & Polypogon monspeliensis (L.) Desf. & Th & COSM & + & + & + & 3 & 100.00 \\
\hline 15 & Senecio glaucus L. & Th & ME+SA-SI+IR-TR & + & + & + & 3 & 100.00 \\
\hline 16 & Setaria verticillata (L.) P. Beauv. & Th & COSM & + & + & + & 3 & 100.00 \\
\hline 17 & Sisymbrium irio L. & Th & ME+IR-TR+ER-SR & + & + & + & 3 & 100.00 \\
\hline 18 & Trifolium resupinatum $\mathrm{L}$. & Th & $\mathrm{ME}+\mathrm{IR}-\mathrm{TR}+\mathrm{ER}-\mathrm{SR}$ & + & + & + & 3 & 100.00 \\
\hline 19 & Vicia sativa $\mathrm{L}$. & Th & ME+IR-TR+ER-SR & + & + & + & 2 & 100.00 \\
\hline 20 & Ammi majus L. & Th & ME+IR-TR+ER-SR & + & + & - & 2 & 66.66 \\
\hline 21 & Atriplex prostrata DC. & Th & ME+ER-SR+IR-TR & + & + & - & 2 & 66.66 \\
\hline 22 & Avena fatua $\mathrm{L}$. & Th & PAL & + & + & - & 2 & 66.66 \\
\hline 23 & Bassia indica (Wight) A.J. Scott. & Th & S-Z+IR-TR & + & + & - & 2 & 66.66 \\
\hline 24 & Bidens pilosa L. & Th & PAN & + & - & + & 2 & 66.66 \\
\hline 25 & Capsella bursa-pastoris L. Medik. & Th & COSM & - & + & + & 2 & 66.66 \\
\hline 26 & Euphorbia peplus L. & Th & ER-SR+ME+IR-TR & - & + & + & 2 & 66.66 \\
\hline 27 & Hordeum marinum Huds. & Th & ME+IR-TR+ER-SR & + & - & + & 2 & 66.66 \\
\hline 28 & Lathyrus hirsuta L. & Th & $\mathrm{ME}+\mathrm{JR}-\mathrm{TR}+\mathrm{ER}-\mathrm{SR}$ & - & + & + & 2 & 66.66 \\
\hline 29 & Lepidium sativum L. & Th & ME & + & - & + & 2 & 66.66 \\
\hline 30 & Poa anпua L. & $\mathrm{Th}$ & COSM & - & + & + & 2 & 66.66 \\
\hline 31 & Ranunculus sceleratus L. & Th & ME+IR-TR+ER-SR & + & + & - & 2 & 66.66 \\
\hline 32 & Senecio vulgaris L. & Th & $\mathrm{ME}+\mathrm{ER}-\mathrm{SR}+\mathrm{IR}-\mathrm{TR}$ & + & + & - & 2 & 66.66 \\
\hline 33 & Torilis arvensis (Huds.) Link & Th & ME+IR-TR+ER-SR & + & - & + & 2 & 66.66 \\
\hline 34 & Urtica urens L. & Th & ME+ER-SR+IR-TR & + & - & + & 2 & 66.66 \\
\hline 35 & Aegilops bicornis (Forssk.) Jaub. \& Spach & Th & $\mathrm{ME}+\mathrm{SA}-\mathrm{SI}$ & + & - & - & 1 & 33.33 \\
\hline 36 & Anthemis pseudocotula Boiss. & Th & ME & + & - & - & 1 & 33.33 \\
\hline 37 & Brassica nigra (L.) Koch & Th & COSM & - & + & - & 1 & 33.33 \\
\hline 38 & Brassica rapa $\mathrm{L}$. & Th & Cult. & - & + & - & 1 & 33.33 \\
\hline 39 & Brassica tournefortii Gouan & Th & ME+IR-TR+SA-SI & - & + & - & 1 & 33.33 \\
\hline 40 & Bromus diandrus Roth & Th & ME & + & - & - & 1 & 33.33 \\
\hline 41 & Cakile maritima Scop. & Th & $\mathrm{ME}+\mathrm{ER}-\mathrm{SR}$ & + & - & - & 1 & 33.33 \\
\hline 42 & Cenchrus biflorus Roxb. & Th & NEO & - & - & + & 1 & 33.33 \\
\hline 43 & Cuscuta pedicellata Ledeb. & $\mathrm{P}$ & S-Z+SA-SI+IR-TR & - & + & - & 1 & 33.33 \\
\hline 44 & Emex spinosa (L.) Campd. & Th & $\mathrm{ME}+\mathrm{SA}-\mathrm{SI}$ & - & - & + & 1 & 33.33 \\
\hline 45 & Euphorbia helioscopia L. & Th & ME+IR-TR+SA-SI & - & - & + & 1 & 33.33 \\
\hline 46 & Lactuca serriola L. & Th & ME+IR-TR+ER-SR & - & - & + & 1 & 33.33 \\
\hline 47 & Lamium amplexicaula L. & Th & ME+IR-TR+ER-SR & - & - & + & 1 & 33.33 \\
\hline 48 & Lathyrus aphaca L. & Th & $\mathrm{ME}+\mathrm{IR}-\mathrm{TR}+\mathrm{ER}-\mathrm{SR}$ & - & - & + & 1 & 33.33 \\
\hline 49 & Lathyrus pseudocicera Pomp. & Th & ME & - & - & + & 1 & 33.33 \\
\hline 50 & Lolium multiflorum Lam. & Th & $\mathrm{ME}+\mathrm{ER}-\mathrm{SR}+\mathrm{IR}-\mathrm{TR}$ & - & + & - & 1 & 33.33 \\
\hline 51 & Mesembryanthemum crystallinum L. & Th & ME+ER-SR & + & - & - & 1 & 33.33 \\
\hline 52 & Mesembryanthemum nodiflorum L. & Th & $\mathrm{ME}+\mathrm{SA}-\mathrm{SI}+\mathrm{ER}-\mathrm{SR}$ & + & - & - & 1 & 33.33 \\
\hline 53 & Orobanche crenata Forssk. & $\mathrm{P}$ & ME+IR-TR & - & + & - & 1 & 33.33 \\
\hline 54 & Parapholis incurva (L.) C.E. Hubb. & Th & ME+IR-TR+ER-SR & + & - & - & 1 & 33.33 \\
\hline 55 & Pennisetum glaucum (L.) R. Br. & Th & PAL & - & - & + & 1 & 33.33 \\
\hline 56 & Reichardia tingitana (L.) Roth & Th & $\mathrm{ME}+\mathrm{SA}-\mathrm{SI}+\mathrm{IR}-\mathrm{TR}$ & + & - & - & 1 & 33.33 \\
\hline 57 & Schismus barbatus (L.) Thell. & Th & ME+IR-TR+SA-SI & - & + & - & 1 & 33.33 \\
\hline 58 & Setaria viridis (L.) P. Beauv. & Th & PAL & + & - & - & 1 & 33.33 \\
\hline 59 & Silene rubella $\mathrm{L}$. & Th & PAL & - & - & + & 1 & 33.33 \\
\hline
\end{tabular}


Table (1). Continued.

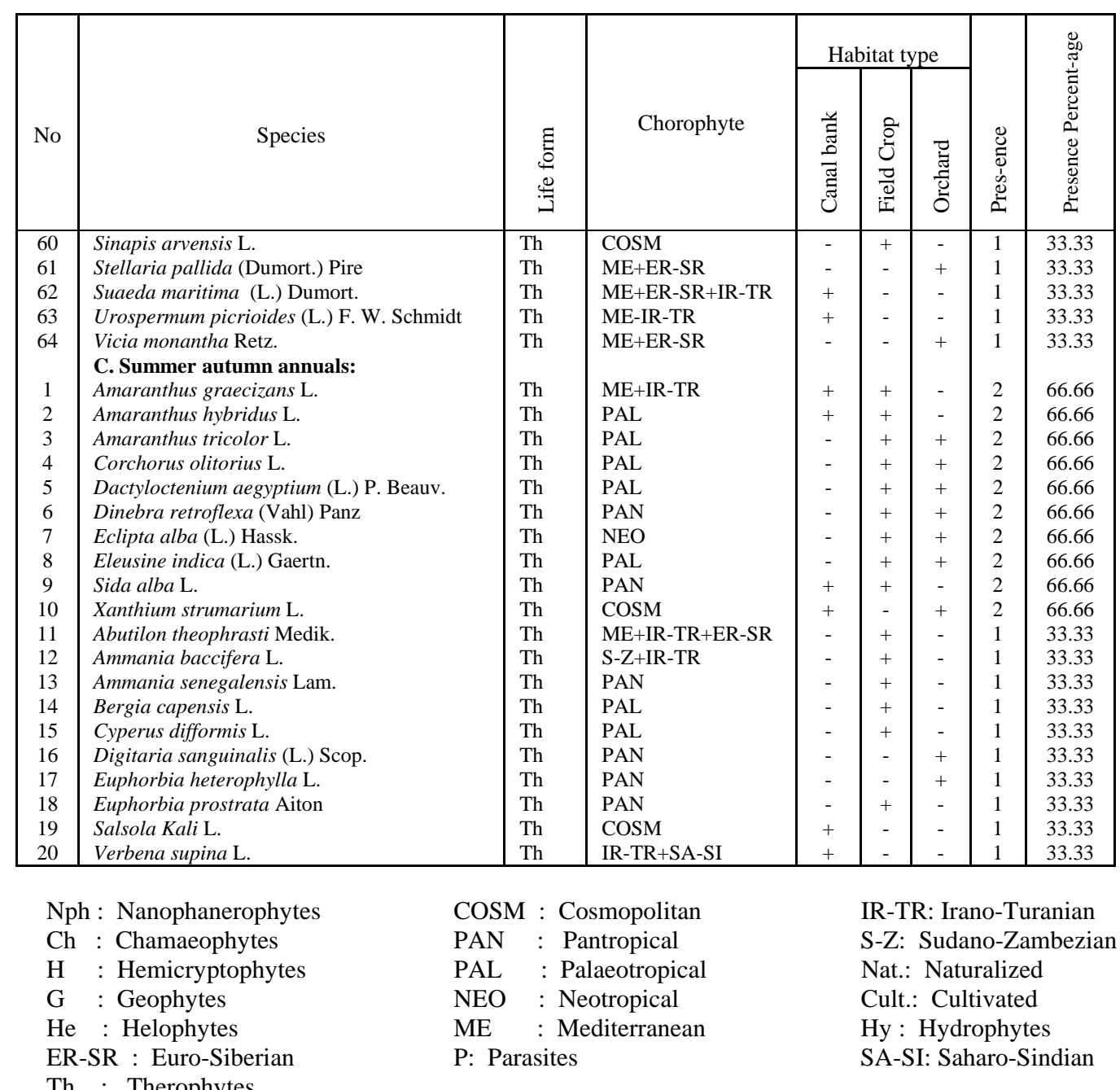

\section{Plant life-span}

According to the duration or life-span, the weeds growing in the area of study can be distinguished into three major groups: annuals, biennials and perennials. As mentioned before, the total recorded species in the study area are 171 taxa classified into 98 annuals (57.31\%), 8 biennials (4.68\%) and 65 perennials (38.01\%) (Fig. 2a).

In the canal and canal bank habitat, 130 species are recorded including: 64 perennials (49.23\%), 6 biennials (4.61\%) and 60 annuals (46.15\%) (Fig. 2b). In the field crop-orchard habitat; 91 species were recorded which classified into: 20 perennials (21.98\%), 6 biennials 
(6.59\%) and 65 annuals (71.43\%) (Fig. 2c). In the orchard habitat, 83 species are recorded which categorised into: 19 perennials (22.89\%), 3 biennials (3.61\%) and 61 annuals (73.49\%) (Fig. 2d).

It is interesting to denote that, the canal and canal bank is floristically the richest habitat in the study area followed by the field crop habitat type and then the orchardha type. It is also obvious that, the annuals are the most frequent species in the different habitats followed by the perennial species and then the less frequent biennial species.
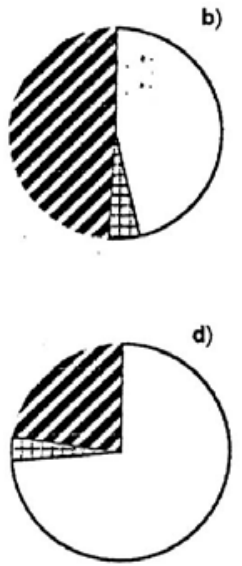

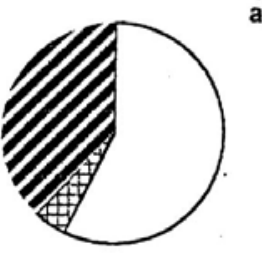

a)
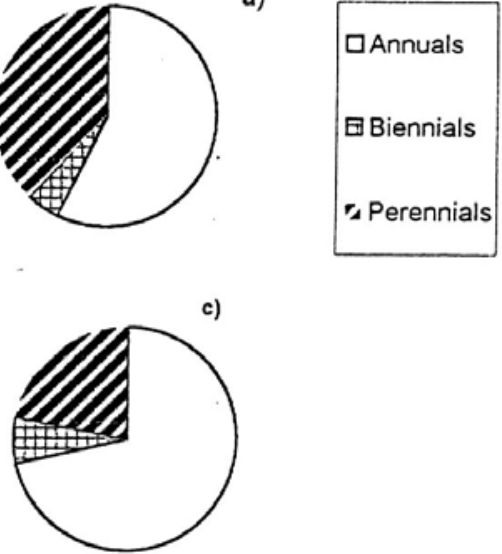

Fig. 2. Plant life span spectra: a. The total recorded species in the study area, b. Canal and canal bank habitat, c. Field crops habitat and d. Orchard habitat.

\section{Plant life-forms}

In the study area, the recorded species (171) are grouped under 6 types of life-forms according to Raunkiaer (1934) as follows: therophytes (103 species $=60.23 \%)$, cryptophytes (38 species $=22.22 \%)$, chamaeophytes $(13$ species $=7.60 \%)$, hemicryptophytes $(12$ species $=7.02 \%)$, nanophanerophytes $(3$ species $=1.75 \%)$ and parasites $(2$ species $=1.17 \%)($ Fig. 3a).

It is evident that, the relative percentages of the life-form spectra vary from one habitat type to the other (Fig. 3a,b,c and d). In the canal bank habitat, the recorded species (130) are classified into the following 5 types of life-forms: therophytes $(51.54 \%)$, cryptophytes (28.46\%), hemicryptophytes (7.69\%), chamaeophytes (10\%) and nanophanerophytes (2.31\%). In the habitat of field crop, the recorded species (91) are grouped into the following life-forms: therophytes (75.82\%), cryptophytes (15.38\%), hemicryptophytes (3.3\%), chamaeophytes (2.20\%), nanophanerophytes $(1.10 \%)$ and parasites $(2.20 \%)$. In the orchard habitat, five types of life-forms are distinguished, these are:therophytes (78.31\%), 
cryptophytes (14.46\%), hemicryptophytes (3.61\%), chamaeophytes (2.41\%) and nanophanerophytes (1.20\%).

It is worth to mention that, the life-form spectrum in all habitat types of the study area is mainly represented by therophytes and partly by cryptophytes, hemicryptophytes and chamaeophytes with a neglibible values for both of nanophanerophytes and parasites. The later life-form is completely missed in both of canal bank and orchard habitat types.

b)

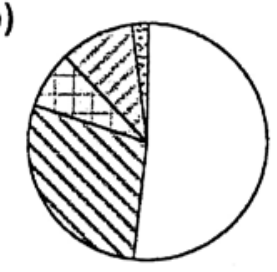

d)

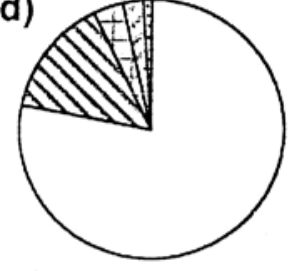

a)

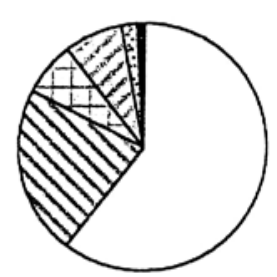

c)

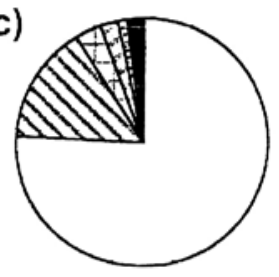

Therophytes

QCryptophytes

G Hemicryptophytes

๑Chamaéophyres

ఐNanophanerphytes

- Parasites

Fig. 3. Plant life forms spectra: a. The total recorded species in the study area, b. Canal and canal bank habitat, c. Field crops habitat and d. Orchard habitat.

\section{Floristic analysis}

The total number of flowering plant species surveyed in the present study is 171 belonging to 128 genera and related to 44 families (Table 2). Gramineae (36 species), Compositae (21), Chenopodiaceae (15), Leguminosae (13) and Cruciferae (11) are represented collectively by 96 species or about $56.14 \%$ of the total number of the recorded species. Cyperaceae (8 species), Polygonaceae (6 species), Amaranthaceae (5 species), Euphorbiaceae and Malvaceae (4 species each) comprise $15.79 \%$ of the total number of species. Caryophyllaceae, Convolvulaceae, Juncaceae and Umbelliferae are represented by 3 species each. The remaining families (30) are either represented by 2 or one species.

Floristically and as shown in Table (2), the most common chorotypes of the Gramineae are: Palaeotropical (8 species), Pantropical (7 species), pluriregional (6 species), biregional and Cosmopolitan (4 species each). In Compositae the most common floristic elements are: pluriregional (6 species), biregional (4 species), Cosmopolitan and Neotropical (3 species each). The most abundant floristic elements in Chenopodiaceae are: Cosmopolitan and pluriregional (5 species each) and biregional (4 species). In Leguminosae, the most common elements are: pluriregional (6 species) and Palaeotropical (3 species), while in Crucifere; Cosmopolitan and pluriregional are the most abundant floristic elements (4 species each). Other families (with less than 10 species each) comprise different types of floristic elements which are generally represented by a few number of species. 
Table (2). The principal floristic categories of the families of the Damietta Province. Abbreviations (see table 1).

\begin{tabular}{|c|c|c|c|c|c|c|c|c|c|c|c|c|c|c|c|c|}
\hline '7[一⿻上丨 & & & & & & & & & $\neg$ & & & & -1 & & & \\
\hline $\mathrm{Z}=\mathrm{s}$ & & & & & & & & & & & & & & & & \\
\hline эฺ̣шәрияี & & & & & & & - & & & & & & & & & \\
\hline IS-vS & & & & & & & & & & & & & $\rightarrow$ & & & \\
\hline HW & & & & & & & $-\infty$ & & - & & & & $\mathrm{a}$ & - & $\rightarrow$ & \\
\hline $\mathrm{T} V \mathrm{~d} \leftarrow$ 国 & & & & & & & & -1 & & -1 & & & $\mathrm{~N}$ & & & \\
\hline [вuotిa.ı!g & & -1 & & -1 & $\rightarrow$ & & $+\theta$ & & - & - & & & + & N & s. & $-\rightarrow-1$ \\
\hline [вuoţిa. - & - & - & & & - & & 0 & & + & & a & 0 & 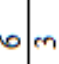 & - & 。 & - \\
\hline OAN & & & & - & & & $m$ & & & & & & $\rightarrow$ & & & \\
\hline TVd & & 2 & & & - & & - & & & $\rightarrow$ & & & $\infty$ & m & $?$ & - \\
\hline $\mathrm{NVd}$ & & - & - & & & & - & & & + & $N$ & & $r$ & & & $\rightarrow-\rightarrow$ \\
\hline WSOD & & & & & & & & & + & $\rightarrow$ & & & + & & $\rightarrow \rightarrow$ & \\
\hline sạ̣ads & & & & & & & & & $\exists$ & $\infty$ & t & c & 잉 & m & & $\mathrm{ata}$ \\
\hline 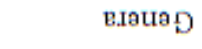 & 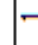 & & & & & & $\infty$ & & $\infty$ & $m$ & - & de & en & $\infty$ & $\infty$ & $-1+$ \\
\hline 窟 & $\frac{5}{7}$ & 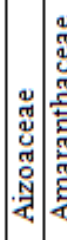 & 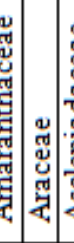 & 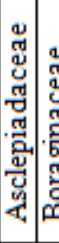 & 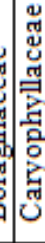 & 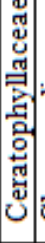 & 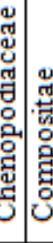 & 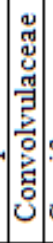 & 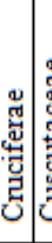 & 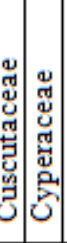 & \begin{tabular}{|l|l} 
& \\
& \\
0 \\
0
\end{tabular} & 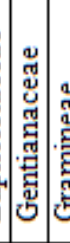 & 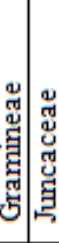 & 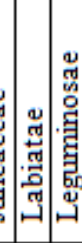 & 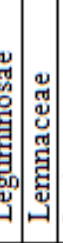 & 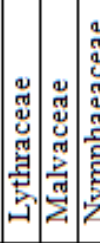 \\
\hline
\end{tabular}


Table (2). Continued.

\begin{tabular}{|c|c|c|c|c|c|c|c|c|c|c|c|c|c|c|c|c|c|c|c|c|}
\hline '7[nכ 28 & & & & & & & & & & & & & & & & & & & & 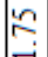 \\
\hline $\mathrm{Z}^{-\mathrm{S}}$ & -1 & & & & & & & & & & & & & & & & & & & . \\
\hline эฺ̣шәри'马 & & & & & & & & & & & & & & & & & & & & 垈 \\
\hline IS-VS & & & & & & & & & & & & & & & & & & & & 年 \\
\hline 百W & & & & & 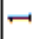 & & & & & & & & & & & & & - & & กิ \\
\hline$T V d \leftarrow$ 互W & & & & & & & & & & & & & & & & & & & & m \\
\hline 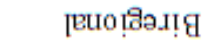 & & - & & & & $\sim$ & & & & & & & -1 & & & & & -1 & & m. \\
\hline [Euo ţa & & & & & & $\rightarrow$ & & & & $\rightarrow$ & & & -1 & & & $m$ & -1 & & & 导 \\
\hline OIN & & & & & & & - & & & & & & & & & & & & b & 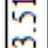 \\
\hline $\mathrm{TVd}$ & & & & & & $m$ & & & & & & & & & & & & & A & $\begin{array}{l}\infty \\
\sim \\
\sim\end{array}$ \\
\hline $\mathrm{NVd}$ & & & & & & & & & & & & & & -1 & -1 & & & - & & ปู \\
\hline WSOD & & & - & - & & & & - & $N$ & & - & -1 & & & & & & & & $m$ \\
\hline sepads & -1 & -1 & - & - & - & 0 & - & - & $N$ & -1 & - & -1 & $N$ & - & -1 & $m$. & -1 & N. & $\equiv$ & \\
\hline R.auอ & -1 & - & - & 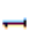 & - & t & $\neg$ & - & $\neg$ & - & - & - & -1 & - & -1 & $m$ & -1 & $2-$ & $\stackrel{\infty}{\stackrel{c}{c}}$ & \\
\hline 壱 & 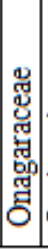 & 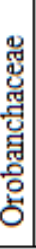 & 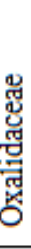 & 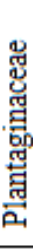 & 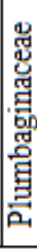 & 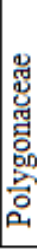 & 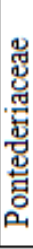 & 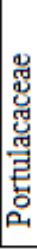 & 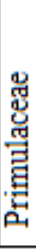 & 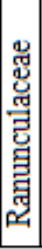 & 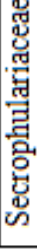 & 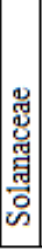 & 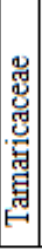 & 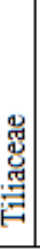 & 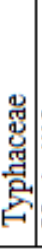 & 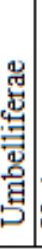 & 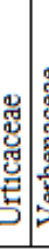 & 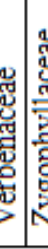 & 중 & 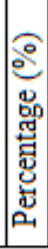 \\
\hline
\end{tabular}


The floristic analysis of the study area as shown in Table 3 reveals that, 81 species or about $47.37 \%$ of the total recorded species are Mediterranean taxa. These taxa are either pluriregional $(46$ species $=26.90 \%)$, biregional $(26$ species $=15.20 \%)$ or monoregional $(9$ species $=5.26 \%$ ). It has been also found that, 77 species or about $45.03 \%$ of the total number of recorded species are either Cosmopolitan (16.37\%), Palaeotropical (12.86\%), Pantropical (12.28\%) or Neotropical (3.51\%). The other floristic categories are poorly represented where each chorotype is represented by a few number of species. In general, the Cosmopolitan, Pantropical, Palaeotropical and Neotropical elements are obviously comparable in all habitats of the study area (Table 3). While, the Mediterranean element is highly represented in canal bank habitat (41 species), followed by the field crop habitat (36 species) and then the orchard habitat type (26 species). Some floristic categories are missed in different habitat types.

Table (3). Number of species and percentage of various floristic categories of the different habitats. Abbreviations (see table 1).

\begin{tabular}{|c|c|c|c|c|c|c|c|c|c|}
\hline \multirow{3}{*}{ No. } & \multirow{3}{*}{ Floristic category } & \multirow{2}{*}{\multicolumn{2}{|c|}{ Total area }} & \multicolumn{6}{|c|}{ Habitat type } \\
\hline & & & & \multicolumn{2}{|c|}{ Orchards } & \multicolumn{2}{|c|}{ Field Crops } & \multicolumn{2}{|c|}{$\begin{array}{c}\text { Canal and } \\
\text { drain banks }\end{array}$} \\
\hline & & No. & $\%$ & No. & $\%$ & No. & $\%$ & No. & $\%$ \\
\hline 1 & COSM & 28 & 16.37 & 19 & 22.89 & 22 & 24.17 & 24 & 18.46 \\
\hline 2 & PAN & 21 & 12.28 & 12 & 14.46 & 12 & 13.19 & 13 & 10.00 \\
\hline 3 & PAL & 22 & 12.86 & 10 & 12.05 & 13 & 14.29 & 19 & 14.62 \\
\hline 4 & $\mathrm{NEO}$ & 6 & 3.51 & 4 & 4.82 & 3 & 3.30 & 4 & 3.08 \\
\hline 5 & $\mathrm{ME}+\mathrm{IR}+\mathrm{TR}+\mathrm{ER}-\mathrm{SR}$ & 33 & 19.30 & 18 & 21.69 & 21 & 23.08 & 23 & 17.69 \\
\hline 6 & ME + IR-TR+SA-SI & 10 & 5.85 & 3 & 3.62 & 5 & 5.49 & 8 & 6.15 \\
\hline 7 & $\mathrm{ME}+\mathrm{SA}-\mathrm{SI}+\mathrm{ER}-\mathrm{SR}$ & 3 & 1.75 & 1 & 1.20 & - & - & 3 & 2.31 \\
\hline 8 & SA-SI + S-Z + IR - TR & 1 & 0.59 & - & - & 1 & 1.10 & - & - \\
\hline 9 & $\mathrm{ME}+\mathrm{IR}-\mathrm{TR}$ & 10 & 5.85 & 5 & 6.02 & 6 & 6.59 & 8 & 6.15 \\
\hline 10 & $\mathrm{ME}+\mathrm{ER}-\mathrm{SR}$ & 9 & 5.26 & 5 & 6.02 & 3 & 3.30 & 7 & 5.38 \\
\hline 11 & $\mathrm{ME}+\mathrm{PAL}$ & 4 & 2.34 & - & - & - & - & 3 & 2.31 \\
\hline 12 & $\mathrm{ME}+\mathrm{SA}-\mathrm{SI}$ & 3 & 1.75 & 1 & 1.20 & - & - & 2 & 1.54 \\
\hline 13 & SA - SI + S-Z & 3 & 1.75 & 1 & 1.20 & 1 & 1.10 & 2 & 1.54 \\
\hline 14 & $\mathrm{~S}-\mathrm{Z}+\mathrm{IR}-\mathrm{TR}$ & 2 & 1.17 & - & - & 2 & 2.20 & 1 & 0.77 \\
\hline 15 & $\mathrm{SA}-\mathrm{SI}+\mathrm{IR}-\mathrm{TR}$ & 1 & 0.59 & - & - & - & - & 1 & 0.77 \\
\hline 16 & $\mathrm{ME}$ & 9 & 5.26 & 3 & 3.62 & 1 & 1.10 & 7 & 5.38 \\
\hline 17 & $\mathrm{SA}-\mathrm{SI}$ & 1 & 0.59 & - & - & - & - & 1 & 0.77 \\
\hline 18 & Endemic & 1 & 0.59 & 1 & 1.20 & - & - & 1 & 0.77 \\
\hline 19 & S-Z & 1 & 0.59 & - & - & - & - & 1 & 0.77 \\
\hline \multirow[t]{2}{*}{20} & Cult. \& Nat. & 3 & 1.75 & - & - & 1 & 1.10 & 2 & 1.54 \\
\hline & Total & 171 & 100 & 83 & 100 & 91 & 100 & 130 & 100 \\
\hline
\end{tabular}

\section{Discussion}

The present study in Damietta area reveals that, the natural plant wealth of the area is composed of 171 species belonging to 128 genera grouped into 44 families. Gramineae (36 species), Compositae (21 species), Chenopodiaceae (15 species), Leguminosae (13 species) and Cruciferae (11 species) are the major families contributing collectively with about $56.14 \%$ of the total recorded species. This indicate that, these five families are leading taxa and 
constitute the main bulk of the flora in the study area. This agrees more or less with findings of Quézel (1978) concerning the floristic structure of the Mediterranean Africa, Mashaly (1987) concerning the floristic studies of Dakahlia-Damietta region and Shalaby (1995) on plant life at Kafr El-Sheikh province.

The recorded species (171 species) are classified into 65 perennial species (38.01\%), 8 biennial species (4.68\%) and 98 annual species (57.31\%). The dominance of annuals could be attributed to the fact that, annuals have higher reproductive capacity and ecological, morphological and genetic plasticity under high levels of disturbance (e.g., agricultural practices; Grime, 1979). Shaltout and El-Fahar (1991) stated that, in cultivated crops, the predominance of annuals (>70\%) in the weed communities in the Nile Delta is mainly related to the niche coincidence of both weeds and host crops. Raunkiaer (1934) designated the Mediterrancean climate type as a "therophyte climate" because of the high percentage (more than $50 \%$ of the total species) of this life form in several Mediterranean floras (Raven, 1971). Plant life forms result from evolved adaptation to environment and climate (Kershaw, 1973). In the present study, the life form spectra indicated that, the therophytes are the most frequent type followed by cryptophytes. This trend is similar to spectra reported for canal bank habitat in the middle Delta region (El-Sheikh, 1989) field crop habitat in Kafr El-Sheikh Province (Shalaby, 1995) and orchard habitat in the middle Delta (El-Kady et al., 1999).

From the phytogeographical point of view, Egypt is the meeting point of floristic elements belonging to at least four phytogeographical regions: the African Sudano-Zambesian, the Asiatic Irano-Turanian, the Afro-Asiatic Sahro-Sindian and the Euro Afro-Asiatic Mediterranean (El-Hadidi, 1993). In the present study, the Mediterranean taxa in Damietta area are represented by a relatively higher percentage of plant species (47.37\%). This was confirmed by El-Demerdash et al. (1990) and Shalaby (1995). The other floristic elements such as Cosmopolitan, Pantropical, Palaeotropical, Irano-Turanian, Saharo-Sindian, EuroSiberian Neotropical and Sudano-Zambezian elements are represented by varying number of species, reflecting their different capability to penetrate the region. This can be attributed to the influence of man and the history of agriculture in the study area.

Sonchus macrocarpus is known to be endemic in the Nile Delta region. It was firstly collected from the cultivated lands of Mansoura-Damietta region (Boulos and Jeffrey 1969), and recently by Mashaly (1987) from the same area and by Shalaby (1995) in Kafr El-Sheikh Province.

The present study favours that, the flora of Damietta area is mainly belonging to the Mediterranean Territory. This opinion is based on the findings in different directions such as: ecoclimatic condition, life-span, life-form spectra and chorological categories.

\section{References}

Abd El-Ghani, M.M. \& Amer, A.M. 1990. Studies on weed assemblages in Croplands, Egypt. I. Broad bean fields. Egypt. J. Bot. 33(1):15-30.

Ayyad, M.A.; Abdel-Razik, M. \& Mehanna, A. 1983. Climatic and vegetation gradients in the Mediterranean desert of Egypt. Pre-report of the Mediterranean Bioclimatology Symposium, Montpelier (France, 18-May pp. III-I-III-2-14).

Boulos, L. 1995. Flora of Egypt, Checklist. Al Hadara Publishing, Cairo, Egypt. 283 pp.

Boulos, L. \& Jeffrey, C. 1969. Sonchus gigas and S. macrocarpus (Comp.) Taxon 18:348-349.

Davis, P.H. ed. 1965-1985. Flora of Turkey and the east Aegean Islands. Vols. 1-9. Edinburgh Univ. Press.

El-Demerdash, M.A., Hosni, H.A. \& Al-Ashri, N. 1997. Distribution of the weed communities in the North East Nile Delta, Egypt. Feddes Repertorium 108(3-4):219-232. 
El-Demerdash, M.A., Zahran, M.A. and Serag, M.S. 1990. On the Ecology of the Deltaic Mediterranean Coastal Land, Egypt. III. The Habitat of salt marshes of Damietta-Port Said coastal region. Arab Gulf J. Scient. Res. 8(3):103-119.

El-Fahar, A.R. 2000. Weed communities of Date Palm orchards in northern Egypt. The first International Conference on Biological Sciences. 7-8 May, Tanta Univ., Tanta, Egypt: 301-314

El-Halawany, E.F. 2000. Flora and vegetation of Date Palm orchards in the Nile Delta Egypt. The first International Conference on Biological Sciences, 7-8 May Tanta Univ., Tanta, Egypt:266-283

El-Kady, H.F.; Shaltout, K.H. \& Mouss, M.T. 1999. Diversity of weed communities in the common orchards of the Nile Delta. J. Union Arab Biol. Cairo 9(B):149-168.

El-Sheikh, M.A. 1989. A study of the vegetation environmental relationships of the canal banks of Middle Delta region. M.Sc. Thesis, Fac. Sci., Tanta Univ., Tanta, Egypt 139 pp.

Grime, J.P. 1979. Plant Strategies and Vegetation Processes. John Wiley \& Sons. 222 pp.

Imam, M. \& Kosinova, J. 1972. Studies on the weed flora of cultivated land in Egypt. 2. Weeds of rice fields. Bot. Jahrb. Syst. Stuttgart 92:90-107.

Kershaw, K.A. 1973. Ouantitative and dynamic plant ecology. The English Language Book Society and Edward Arnold Publishers, London, 308 p.

Kosinova, J. 1974a. Studies on the weed flora of cultivated land in Egypt. 3. Distributional types, Bot. Jahrb. Syst., 94: 449-458.

Kosinova, J. 1975. Weed communities of winter crops in Egypt. Preslia, 47: 58-74.

Mashaly, I.A. (1987): Ecological and floristic studies of Dakahlia-Damietta region, Mansoura Univ., Fac. Sci., Ph.D. Thesis.

Meikle, R.D. (1977 \& 1985): Flora of Cyprus. Vol. 1\&2. Bentham-Maxon Trust, Royal Botanic Gardens, Kew.

Quezel, P. (1978). Analysis of the flora of Mediterranean and Sahran Africa: Phytogeography of Africa. Ann. Missour. Bot. Gard., 65: 479-534.

Raunkiaer, C. (1934). The life-forms of plants and statistical plant geography. Translated by Carrter, Fausboll and Tansley Oxford Univ. Press, London.

Raven, P. (1971): Relationships between Mediterranean floras In Davis, P.H., Harper, P.C. \& Hedge, I.C. (eds.). Plant life in south-west Asia. J. Botanical Society of Edinburgh, Edinburgh: 119-134.

Shalaby, M.A. (1995): Studies on plant life at Kafr El Sheikh Province-Egypt. M.Sc. Thesis Fac. of Agriculture Kafr El Sheikh, Tanta Univ.

Shaltout, K.H. and El-Fahar, R.A. (1991): Diversity and phenology of weed communities in the Nile Delta region. Jour. Veg. Sci., 2: 385-390.

Shaltout, K.H., Sharaf El-Din, A. and El-Sheikh, M.A. (1994). Species richness and phenology of vegetation along irrigation canals and drains in the Nile Delta, Egypt. Vegetatio 112:35-43.

Sheded, M. and Turki, Z. (2000): Distribution of the weed communities in the south Nile Delta Egypt. The first International Conference on Biological Sciences. 7-8 May Tanta Univ. Tanta, Egypt: 171-182.

Simpson, N.D. (1932): A report on the weed flora of the irrigation channels in Egypt. Gov. Press. Cairo.

Tackholm, V. (1974): Students’ Flora of Egypt. $2^{\text {nd }}$ edition Cairo Univ. Press.

Zaki, M.A. and Mashaly, I.A. (1992): Seasonal aspects of the flora of the field crops, Nile Delta, Egypt. Mansoura Sci. Bull., 19: 187-202. 